\title{
Solving Liouville-type Problems on Manifolds with Poincaré-Sobolev Inequality by Broadening $q$-Energy from Finite to Infinite
}

\author{
Lina $\mathrm{Wu}^{1}$ \\ ${ }^{1}$ Department of Mathematics, Borough of Manhattan Community College-The City University of New York, New York, \\ NY 10007, USA \\ Correspondence: Lina Wu, Department of Mathematics, Borough of Manhattan Community College-The City University \\ of New York, New York, NY 10007, USA. Tel: 1-212-346-8601. Fax: 1-212-748-7459. E-mail: lwu@ bmcc.cuny.edu
}

Received: May 22, 2017 Accepted: June 12, 2017 Online Published: June 29, 2017

doi:10.5539/jmr.v9n4p1 URL: https://doi.org/10.5539/jmr.v9n4p1

\begin{abstract}
The aim of this article is to investigate Liouville-type problems on complete non-compact Riemannian manifolds with Poincaré-Sobolev Inequality. Two significant technical breakthroughs are demonstrated in research findings. The first breakthrough is an extension from non-flat manifolds with non-negative Ricci curvatures to curved manifolds with Ricci curvatures varying among negative values, zero, and positive values. Poincaré-Sobolev Inequality has been applied to overcome difficulties of an extension on manifolds. Poincaré-Sobolev Inequality has offered a special structure on curved manifolds with a mix of Ricci curvature signs. The second breakthrough is a generalization of $q$-energy from finite to infinite. At this point, a technique of $p$-balanced growth has been introduced to overcome difficulties of broadening from finite $q$-energy in $L^{q}$ spaces to infinite $q$-energy in non- $L^{q}$ spaces. An innovative computational method and new estimation techniques are illustrated. At the end of this article, Liouville-type results including vanishing properties for differential forms and constancy properties for differential maps have been verified on manifolds with Poincaré-Sobolev Inequality approaching to infinite $q$-energy growth.
\end{abstract}

Keywords: closed differential forms, finite $q$-energy in $L^{q}$ spaces, Hölder Inequality, $p$-balanced growth, $p$-harmonic maps, Poincaré-Sobolev Inequality, $p$-pseudo-coclosed differential forms, Weitzenböck Bochner Formula

2000 Mathematics Subject Classification 53C40, 53C23, 53C21

\section{Introduction}

The study of Liouville-type problems is to obtain constancy properties for differential forms and differential maps on manifolds equipped with a wide variety of metric structures. It has been one of the most valuable and challenging research topics in the mathematical society. Mathematicians have obtained Liouville-type results as follows:

1. Liouville-type results for differential forms: The first result goes back to Greene and Wu (Greene \& Wu, 1981). In 1981, Greene and $\mathrm{Wu}$ solved Liouville-type problems for harmonic 1-forms and obtained vanishing property for harmonic 1-forms with finite $q$-energy in $L^{q}(1<q<\infty)$ spaces on non-negatively curved manifolds $M$ with Ricci $^{M} \geq 0$. In 2001, Zhang (Zhang, 2001) studied Liouville-type problems for closed and $p$-co-closed differential 1 -forms $(p>1)$ in $L^{q}(0<q<\infty)$ spaces on positively curved manifolds $M$.

2. Liouville-type results for differential maps: In 1976, Schoen and Yau (Schoen \& Yau, 1976) solved Liouville-type problems for harmonic maps and established constancy property for harmonic maps on $M$ with Ricci $^{M} \geq 0$. In 1995 Cheung and Leung (Cheung \& Leung, 1995) showed Liouville Theorems for $p$-harmonic maps $(p \geq 2)$ in $L^{q}(q=p-1)$ spaces on the target spaces of Cartan-Hadamand Manifolds. In 1999, Kawai (Kawai, 1999) derived Liouville Theorems for $p$-harmonic maps $(p \geq 2)$ from $p$-parabolic manifolds to non-positively curved targets. In 2007, Pigola, Rigoli, and Setti (Pigola, Rigoli \& Setti, 2007) studied Liouville Theorems for $p$-harmonic maps $(p \geq 2)$ into non-positively curved manifolds.

The aim of this article is to investigate Liouville-type problems for both differential forms and differential maps on complete non-compact manifolds $M$ in the presence of Poincaré-Sobolev Inequality. It is well-known that any non-flat manifold can be determined by the sign of its curvatures. A curved non-flat manifold can be classified into either a manifold with only one sign of its curvatures (such as a globally positive curved manifold or a globally negative curved manifold) or a manifold with the mixed signs of its curvatures (a combination of locally positive curvatures with locally negative curvatures). The main study of Liouville-type problems on manifolds is to study various manifolds $M$ equipped 
with all possible different metric structures $(M, g)$ such that there exist constancy properties or vanishing properties for differential forms and differential maps on $(M, g)$.

Liouville-type problems on either globally positive curved manifolds or globally negative curved manifolds have been studied by mathematicians for decades. Most research findings have been obtained in $L^{q}$ spaces with finite $q$-energy on curved manifolds with only one sign of curvatures. Many questions are still open. For example, how to solve Liouvilletype problems on curved manifolds with the mixed curvature signs and how to solve Liouville-type problems with infinite $q$-energy in non- $L^{q}$ spaces.

Liouville-type results have been generalized from finite $q$-energy in $L^{q}$ spaces to infinite $q$-energy in non- $L^{q}$ spaces as well as the extended scope of $q$. For example, Liouville-type results such as vanishing properties for harmonic 1-forms with infinite $q$-energy on manifolds with positive semi-definite Ricci curvatures were discovered in 2015 by S.W. Wei and Wu (Wei \& Wu, 2015). Liouville-type results for closed and $p$-pseudo-coclosed differential 1-forms with infinite $q$-energy on manifolds with non-negative Ricci curvatures were obtained in 2016 by Y. $\mathrm{Li}$ and $\mathrm{Wu}(\mathrm{Wu} \& \mathrm{Li}, 2016)$. However, the generalization of $q$-energy in Liouville-type theorems in the past research work had been proven on manifolds with only one sign of curvatures.

The goal of this article is to solve Liouville-type problems on curved manifolds with the mixed curvature signs. Research findings of Liouville-type results on various manifold structures determined by the mixed signs of curvatures are obtained. In particular, vanishing properties for differential forms and constancy properties for differential maps are verified in both finite $q$-energy in $L^{q}$ spaces and infinite $q$-energy in non- $L^{q}$ spaces. Two significant technical breakthroughs are demonstrated. The first breakthrough is an extension from non-flat manifolds with non-negative Ricci curvatures to curved manifolds with Ricci curvatures at any values, which vary among negative values, zero, and positive values. Poincaré-Sobolev Inequality has been applied to overcome difficulties of manifold structure extensions. Poincaré-Sobolev Inequality has offered a special structure on curved manifolds for Ricci curvatures with the mixed signs. It turns out that Poincaré-Sobolev Inequality survives on various manifolds with quite different structures in the large scope of metric changes. The second breakthrough is an extension from finite $q$-energy $L^{q}$ spaces to broader spaces, which include both finite $q$-energy $L^{q}$ spaces and infinite $q$-energy non- $L^{q}$ spaces. At this point, the technique of $p$-balanced growth has been studied to overcome difficulties of the $q$-energy generalization.

As compared with traditional calculation methods used to solve Liouville-type problems in the context of finite $q$-energy, an innovative computational method leading to infinite $q$-energy is demonstrated in this article. Weitzenböck Bochner Formula, Poincaré-Sobolev Inequality, Hölder Inequality, Cauchy-Schwarz Inequality, and Calculus skills as estimation techniques are presented. Weitzenböck Bochner Formula is used at the beginning as the foundation to establish the first inequality regarding integrals of differential forms or differential maps on curved manifolds. After that, an appropriate test function with its power varying in a certain range is carefully selected. Changes of power in this selected test function play an important role to reveal the way how changes of manifold structures have an impact on changes of energy growth rates for differential maps or differential maps. The maximum range of power in this test function determines the maximum scope of energy growth rates. Energy growth rates being too fast or too slow as indicated by too high or too low power in the test function lead to contradictions with the maximum scope of manifolds whose structures are compatible with Poincaré-Sobolev Inequality. At the end, a balance between reasonable energy growth rates and appropriate manifolds supported by Poincaré-Sobolev Inequality is made to obtain Liouville-type results.

\section{Preliminary}

In this section, we first define the concept of $p$-balanced growth for $p>1$, which consists of 5 cases: $p$-finite growth, $p$-mild growth, $p$-obtuse growth, $p$-moderate growth, and $p$-small growth. After that, we give definitions of closed differential forms, $p$-pseudo-coclosed differential forms, and $p$-harmonic maps. At the end of this section, PoincaréSobolev Inequality on a complete non-compact Riemannian manifold is defined.

Throughout this paper, we assume that $M$ is a complete non-compact $n$-dimensional Riemannian manifold and $B\left(x_{0} ; r\right)$ (or $B(r)$ ) is the geodesic ball of radius $r$ centered at a point $x_{0} \in M$. A function or a differential form $f$ is said to be with $p$-balanced growth provided $f$ has one of the following " $p$-finite, $p$-mild, $p$-obtuse, $p$-moderate, and $p$-small" growth where $p>1$. Otherwise, $f$ is said to be with $p$-imbalanced growth (Wei, Li \& Wu, 2008).

Definition 1 A function $f$ has $p$-finite growth (or, simply, is p-finite) if there exists $x_{0} \in M$ such that

$$
\liminf _{r \rightarrow \infty} \frac{1}{r^{p}} \int_{B\left(x_{0} ; r\right)}|f|^{q} d v<\infty
$$

and has p-infinite growth (or, simply, is p-infinite) otherwise.

Definition 2 A function fhas p-mild growth (or, simply, is p-mild) if there exist $x_{0} \in M$, and a strictly increasing sequence 
of $\left\{r_{j}\right\}_{0}^{\infty}$ going to infinity, such that for every $l_{0}>0$, we have:

$$
\sum_{j=l_{0}}^{\infty}\left(\frac{\left(r_{j+1}-r_{j}\right)^{p}}{\int_{B\left(x_{0} ; r_{j+1}\right) \backslash B\left(x_{0} ; r_{j}\right)}|f|^{q} d v}\right)^{\frac{1}{p-1}}=\infty
$$

and has p-severe growth (or, simply, is p-severe) otherwise.

Definition 3 A function f has p-obtuse growth (or, simply, is p-obtuse) if there exists $x_{0} \in M$ such that for every a $>0$, we have:

$$
\int_{a}^{\infty}\left(\frac{1}{\int_{\partial B\left(x_{0} ; r\right)}|f|^{q} d v}\right)^{\frac{1}{p-1}} d r=\infty
$$

and has p-acute growth (or, simply, is p-acute) otherwise.

Definition 4 A function $f$ has $p$-moderate growth (or, simply, is p-moderate) if there exist $x_{0} \in M$, and $F(r) \in \mathcal{F}$, such that

$$
\limsup _{r \rightarrow \infty} \frac{1}{r^{p} F^{p-1}(r)} \int_{B\left(x_{0} ; r\right)}|f|^{q} d v<\infty
$$

and has p-immoderate growth (or, simply, is p-immoderate) otherwise, where

$$
\mathcal{F}=\left\{F:[a, \infty) \rightarrow(0, \infty) \mid \int_{a}^{\infty} \frac{d r}{r F(r)}=\infty\right\}
$$

for some $a>0$. Notice that the functions in $\mathcal{F}$ are not necessarily monotone.

Definition 5 A function $f$ has $p$-small growth (or, simply, is p-small) if there exists $x_{0} \in M$, such that for every $a>0$, we have:

$$
\int_{a}^{\infty}\left(\frac{r}{\int_{B\left(x_{0} ; r\right)}|f|^{q} d v}\right)^{\frac{1}{p-1}} d r=\infty
$$

and has p-large growth (or, simply, is p-large) otherwise.

The above definitions of " $p$-finite, $p$-mild, $p$-obtuse, $p$-moderate, $p$-small" and their counter-parts " $p$-infinite, $p$-severe, $p$-acute, $p$-immoderate, $p$-large" growth depend on $q$, and $q$ will be specified in the context in which the definition is used.

Let $N$ be a complete Riemannian manifold.

Definition 6 For a map $u: M \rightarrow N$, we define the p-energy of $u$ by:

$$
E_{p}(u)=\frac{1}{p} \int_{M}|d u|^{p} d v
$$

where $d v$ is the volume element of $M$ and $p>1$.

Let $\mathcal{A}^{k}(\rho)=C\left(\wedge^{k} T^{*} M \otimes V\right)$ be a space of smooth $k$-forms on $M$ with values in the vector bundle $\rho: V \rightarrow M$, and let $d: \mathcal{A}^{k}(\rho) \rightarrow \mathcal{A}^{k+1}(\rho)$ be the exterior differential operator and let $d^{*}: \mathcal{A}^{k}(\rho) \rightarrow \mathcal{A}^{k-1}(\rho)$ be the exterior differential operator given by $d^{*}=-\sum_{j=1}^{n} i\left(e_{j}\right) \nabla_{e_{j}}$ where $\left\{e_{j}\right\}$ is a local orthonormal frame at $x \in M$, and $i(X)$ is the interior multiplication by $X$ defined as $(i(X) v)\left(Y_{1}, \cdots, Y_{k-1}\right)=v\left(X, Y_{1}, \cdots, Y_{k-1}\right)$ for any $X \in T_{x}(M), v \in \mathcal{A}^{k}(\rho)$ and $Y_{l} \in T_{x}(M), 1 \leq l \leq k-1$ . In particular, if $v \in \mathcal{A}^{1}(\rho), d^{*}$ is also defined by $d^{*} v=-\operatorname{trace} \nabla v=-$ divv. The Hodge Laplacian $\Delta$ is defined on the $V$-valued differential forms by

$$
\Delta=-\left(d d^{*}+d^{*} d\right): \mathcal{A}^{k}(V) \rightarrow \mathcal{A}^{k}(V)
$$

Definition 7 A map $u$ is said to be a p-harmonic map $(p>1)$ if it is a critical point of p-energy functional $E_{p}$. Equivalently, $u$ is a p-harmonic map if and only if the p-tension field

$$
\tau_{p}(u)=\operatorname{div}\left(|\nabla u|^{p-2} \nabla u\right)=-d^{*}\left(|d u|^{p-2} d u\right)=0
$$

In particular, a map $u$ is said to be a harmonic map if $u$ is a p-harmonic map when $p=2$. Equivalently, $u$ is a harmonic map if and only if the 2-tension field

$$
\tau(u)=\operatorname{div}(\nabla u)=-d^{*}(d u)=0
$$

Definition 8 A differential form $\omega$ is said to be closed if $d \omega=0 . \omega$ is said to be p-pseudo-coclosed $(p>1)$ if

$$
d^{*}\left(|\omega|^{p-2} \omega\right)=0
$$


For example, the differential of a $p$-harmonic function is a closed and $p$-pseudo-coclosed 1-form.

Definition 9 A complete non-compact $n$-dimensional Riemannian manifold $M$ is said to support Poincaré-Sobolev Inequality if $M$ satisfies:

$$
S_{\alpha}\left\|\varphi^{2}\right\|_{L_{M}^{1-\alpha}} \leq\|\nabla \varphi\|_{L_{M}^{2}}^{2}
$$

for every compactly supported non-negative smooth function on $M$ (that is, $\varphi \in C_{0}^{\infty}(M)$ and $\varphi \geq 0$ ) and for some positive constant $S_{\alpha}>0$ where

$$
\alpha= \begin{cases}\frac{2}{n} & \text { when } n>2 \\ 0 \leq \alpha<1 & \text { when } n \leq 2 .\end{cases}
$$

The version of Poincaré-Sobolev Inequality given in this paper is obtained by squaring both sides of the standard PoincaréSobolev Inequality (that is, $S\|\varphi\|_{L_{M}^{n-p}} \leq\|\nabla \varphi\|_{L_{M}^{p}}$ ) for constants $S=\sqrt{S_{\alpha}}>0$ and $\alpha=\frac{p}{n}$ and $p=2$ when $n>2$.

\section{Results}

In this section, on complete non-compact Riemannian manifolds $M$ with Poincaré-Sobolev Inequality, lemmas and a sequence of Liouville-type theorems are proven in the following two categories: vanishing properties for differential forms and constancy properties for differential maps.

\subsection{Liouville-type Results of Vanishing Properties for Differential Forms}

Lemma 1 (Wu \& Li, 2016, Lemma 3.2) Suppose a differential 1-form $\omega$ is closed and p-pseudo-coclosed on M. Let $\eta \geq 0$ and $\eta \in C_{0}^{\infty}(M)$ (that is, a compactly supported non-negative smooth function on $M$ ), and $\xi=\eta|\omega|^{m}$. Then for $m \geq p \geq 2$, we have:

$$
\begin{aligned}
& \int_{M} \xi^{2}\langle\Delta \omega, \omega\rangle d v \\
= & \frac{(p-2)(2 m+2-p)}{4} \int_{M} \eta^{2}|\omega|^{2 m-4}\left\langle d|\omega|^{2}, \omega\right\rangle^{2} d v+(p-2) \int_{M} \eta|\omega|^{2 m-2}\left\langle d|\omega|^{2}, \omega\right\rangle\langle d \eta, \omega\rangle d v \\
\geq & -\left.(p-2) \int_{M} \eta|\nabla \eta||\omega|^{2 m}|\nabla| \omega\right|^{2} \mid d v
\end{aligned}
$$

Theorem 1 Let $M$ be a complete non-compact Riemannian manifold satisfying Poincaré-Sobolev Inequality (1) and Ricci $^{M} \geq-k(x)$ where $k(x) \geq 0$ is a continuous function such that

$$
k_{\alpha}: \triangleq\|k(x)\|_{L_{M}^{\frac{1}{\alpha}}}<S_{\alpha}
$$

Let $\omega$ be a closed and p-pseudo-coclosed differential 1-form on $M$ for $p \geq 2$. Then $\omega=0$ if $\omega$ has:

$$
\liminf _{r \rightarrow \infty} \frac{1}{r^{2}} \int_{B(x, r)}|\omega|^{q} d v=0
$$

In particular, $\omega=0$ if $\omega \in L^{q}(M)$ has:

$$
\int_{M}|\omega|^{q} d v<\infty
$$

And $q$ is given by

$$
\max \left\{2 p+2, \frac{2\left(1-\sqrt{1-k_{\alpha} S_{\alpha}^{-1}}\right)}{k_{\alpha} S_{\alpha}^{-1}}\right\}<q<\frac{2\left(1+\sqrt{1-k_{\alpha} S_{\alpha}^{-1}}\right)}{k_{\alpha} S_{\alpha}^{-1}}
$$

proof. Applying Weitzenböck Bochner Formula for $\omega$ on $M$, we have:

$$
\begin{aligned}
\frac{1}{2} \Delta|\omega|^{2} & =\langle\Delta \omega, \omega\rangle+|\nabla \omega|^{2}+\sum_{j=1}^{n} \omega\left(\operatorname{Ricci}^{M}\left(e_{j}\right)\right) \cdot \omega\left(e_{j}\right) \\
& \geq\langle\Delta \omega, \omega\rangle+|\nabla \omega|^{2}-k(x)|\omega|^{2}
\end{aligned}
$$

where $\left\{e_{1}, \cdots e_{n}\right\}$ is a local orthonormal frame on $M$. It follows from (3), and an observation $|\nabla \omega| \geq|\nabla| \omega||$ that:

$$
\langle\Delta \omega, \omega\rangle+|\nabla| \omega||^{2}-k(x)|\omega|^{2}-\frac{1}{2} \Delta|\omega|^{2} \leq 0
$$

We choose a test function $\xi=\eta|\omega|^{m}$ for $m \geq p \geq 2$ where $\eta$ is a rotationally symmetric Lipschitz continuous function $\eta=\eta(x ; s, t), 0<s<t$ satisfying the following properties:

1. $\eta=1$ on $B(x ; s), \eta=0$ off $B(x ; t)$, and $0 \leq \eta \leq 1$ on $B(x ; t) \backslash B(x ; s)$; 
2. $|\nabla \eta| \leq \frac{C_{1}}{t-s}$ a.e. on $M$ where a positive constant $C_{1}>0$ is independent with choices of $s$ and $t$.

Multiplying $\xi^{2}$ on both sides of (4) and applying integration by parts to $-\frac{1}{2} \int_{M} \xi^{2} \Delta|\omega|^{2} d v$ yield:

$$
\int_{M} \xi^{2}\langle\Delta \omega, \omega\rangle d v+\int_{M} \xi^{2}|\nabla| \omega \|^{2} d v-\int_{M} k(x) \xi^{2}|\omega|^{2} d v+\int_{M} \xi\left\langle\nabla \xi, \nabla|\omega|^{2}\right\rangle d v \quad \leq 0
$$

In order to estimate $\int_{M} k(x) \xi^{2}|\omega|^{2} d v$, we apply Hölder inequality for $\int_{M} k(x) \eta^{2}|\omega|^{2 m+2} d v$ and apply Poincaré-Sobolev inequality (1) for $\left(\int_{M}\left(\eta|\omega|^{m+1}\right)^{\frac{2}{1-\alpha}} d v\right)^{1-\alpha}$ with $\varphi=\eta|\omega|^{m+1}$ and use the fact $|\nabla| \omega \mid \|=\frac{\left.|\nabla| \omega\right|^{2} \mid}{2|\omega|}$ to have:

$$
\begin{aligned}
& \int_{M} k(x) \xi^{2}|\omega|^{2} d v \\
& =\int_{M} k(x) \eta^{2}|\omega|^{2 m+2} d v \\
& \leq\left(\int_{M} k(x)^{\frac{1}{\alpha}} d v\right)^{\alpha}\left(\int_{M}\left(\eta|\omega|^{m+1}\right) \frac{2}{1-\alpha} d v\right)^{1-\alpha} \\
& \leq k_{\alpha} S_{\alpha}^{-1} \int_{M}\left|\nabla\left(\eta|\omega|^{m+1}\right)\right|^{2} d v \\
& =k_{\alpha} S_{\alpha}^{-1} \int_{M}\left\{|\omega|^{2 m+2}|\nabla \eta|^{2}+\left.(m+1)^{2} \eta^{2}|\omega|^{2 m}|\nabla| \omega\right|^{2}+2(m+1) \eta|\omega|^{2 m+1}\langle\nabla \eta, \nabla|\omega|\rangle\right\} d v \\
& \leq k_{\alpha} S_{\alpha}^{-1} \int_{M}|\omega|^{2 m+2}|\nabla \eta|^{2} d v+\left.\left.k_{\alpha} S_{\alpha}^{-1} \frac{(m+1)^{2}}{4} \int_{M} \eta^{2}|\omega|^{2 m-2}|\nabla| \omega\right|^{2}\right|^{2} d v \\
& \quad+\left.k_{\alpha} S_{\alpha}^{-1}(m+1) \int_{M} \eta|\nabla \eta \| \omega|^{2 m}|\nabla| \omega\right|^{2} \mid d v
\end{aligned}
$$

By estimating $\int_{M} \xi\left\langle\nabla \xi, \nabla|\omega|^{2}\right\rangle d v$, we have:

$$
\begin{aligned}
& \int_{M} \xi\left\langle\nabla \xi, \nabla|\omega|^{2}\right\rangle d v \\
& =\int_{M} \eta|\omega|^{m}\left\langle\nabla\left(\eta|\omega|^{m}\right), \nabla|\omega|^{2}\right\rangle d v \\
& =\int_{M} \eta|\omega|^{2 m}\left\langle\nabla \eta, \nabla|\omega|^{2}\right\rangle d v+\left.\left.\int_{M} \frac{m}{2} \eta^{2}|\omega|^{2 m-2}|\nabla| \omega\right|^{2}\right|^{2} d v \\
& \geq-\left.\left.\left.\left.\int_{M} \eta|\nabla \eta||\omega|^{2 m}|\nabla| \omega\right|^{2}\left|d v+\int_{M} \frac{m}{2} \eta^{2}\right| \omega\right|^{2 m-2}|\nabla| \omega\right|^{2}\right|^{2} d v
\end{aligned}
$$

By substituting (6),(7), and (2) into (5) for $m \geq p \geq 2$ and calculating $\int_{M} \xi^{2}|\nabla| \omega \|^{2} d v=\left.\left.\frac{1}{4} \int_{M} \eta^{2}|\omega|^{2 m-2}|\nabla| \omega\right|^{2}\right|^{2} d v$, we have:

$$
\begin{aligned}
& -\left.\left.\left.\left.\left.\left(p-1+k_{\alpha} S_{\alpha}^{-1}(m+1)\right) \int_{M} \eta|\nabla \eta \|| \omega\right|^{2 m}|\nabla| \omega\right|^{2}\left|d v+\frac{2 m+1-k_{\alpha} S_{\alpha}^{-1}(m+1)^{2}}{4} \int_{M} \eta^{2}\right| \omega\right|^{2 m-2}|\nabla| \omega\right|^{2}\right|^{2} d v \\
& -k_{\alpha} S_{\alpha}^{-1} \int_{M}|\omega|^{2 m+2}|\nabla \eta|^{2} d v \leq 0
\end{aligned}
$$

where

$$
\frac{2 m+1-k_{\alpha} S_{\alpha}^{-1}(m+1)^{2}}{4}>0
$$

if and only if

$$
\frac{1-\sqrt{1-k_{\alpha} S_{\alpha}^{-1}}}{k_{\alpha} S_{\alpha}^{-1}}-1<m<\frac{1+\sqrt{1-k_{\alpha} S_{\alpha}^{-1}}}{k_{\alpha} S_{\alpha}^{-1}}-1
$$

At the same time, by Hölder inequality, we have:

$$
\left.\int_{B(t) \backslash B(s)} \eta|\nabla \eta \| \omega|^{2 m}|\nabla| \omega\right|^{2} \mid d v \leq\left(\left.\left.\int_{B(t) \backslash B(s)} \eta^{2}|\omega|^{2 m-2}|\nabla| \omega\right|^{2}\right|^{2} d v\right)^{\frac{1}{2}}\left(\int_{B(t) \backslash B(s)}|\nabla \eta|^{2}|\omega|^{2 m+2} d v\right)^{\frac{1}{2}}
$$

Therefore, via (8) and (10) and observing properties of $\eta$, we have:

$$
\begin{aligned}
& \left.\left.\frac{2 m+1-k_{\alpha} S_{\alpha}^{-1}(m+1)^{2}}{4} \int_{B(t)} \eta^{2}|\omega|^{2 m-2}|\nabla| \omega\right|^{2}\right|^{2} d v \\
& \leq k_{\alpha} S_{\alpha}^{-1} \int_{B(t) \backslash B(s)}|\omega|^{2 m+2}|\nabla \eta|^{2} d v \\
& +\left(p-1+k_{\alpha} S_{\alpha}^{-1}(m+1)\right)\left(\left.\left.\int_{B(t) \backslash B(s)} \eta^{2}|\omega|^{2 m-2}|\nabla| \omega\right|^{2}\right|^{2} d v\right)^{\frac{1}{2}}\left(\int_{B(t) \backslash B(s)}|\nabla \eta|^{2}|\omega|^{2 m+2} d v\right)^{\frac{1}{2}}
\end{aligned}
$$

Now, we define:

$$
\begin{aligned}
A_{r} & =\frac{1}{r^{2}} \int_{B(r)}|\omega|^{2 m+2} d v \\
A_{j}: \triangleq A_{r_{j}} & =\frac{1}{r_{j}^{2}} \int_{B\left(r_{j}\right)}|\omega|^{2 m+2} d v \\
Q_{r} & =\left.\left.\int_{B(r)} \eta^{2}|\omega|^{2 m-2}|\nabla| \omega\right|^{2}\right|^{2} d v \\
\eta_{j} & =\eta=\eta\left(x, r_{j}, r_{j+1}\right) \\
Q_{j+1}: \triangleq Q_{r_{j+1}} & =\left.\left.\int_{B\left(r_{j+1}\right)} \eta_{j}^{2}|\omega|^{2 m-2}|\nabla| \omega\right|^{2}\right|^{2} d v
\end{aligned}
$$


where $\left\{r_{j}\right\}$ is a strictly increasing sequence going to infinity. So, by setting $\eta=\eta_{j}, t=r_{j+1}, s=r_{j}$ and observing facts $\eta_{j-1} \leq \eta_{j}, \quad\left|\nabla \eta_{j}\right| \leq \frac{C_{1}}{r_{j+1}-r_{j}}$ for any $j$, we have the following:

$$
C_{2} Q_{j+1} \leq C_{3} \frac{r_{j+1}^{2} A_{j+1}-r_{j}^{2} A_{j}}{\left(r_{j+1}-r_{j}\right)^{2}}+C_{4}\left(Q_{j+1}-Q_{j}\right)^{\frac{1}{2}} \frac{\left(r_{j+1}^{2} A_{j+1}-r_{j}^{2} A_{j}\right)^{\frac{1}{2}}}{r_{j+1}-r_{j}}
$$

where

$$
\begin{aligned}
& C_{2}=\frac{2 m+1-k_{\alpha} S_{\alpha}^{-1}(m+1)^{2}}{4}>0 \\
& C_{3}=k_{\alpha} S_{\alpha}^{-1} C_{1}^{2}>0 \\
& C_{4}=\left(p-1+k_{\alpha} S_{\alpha}^{-1}(m+1)\right) C_{1}>0
\end{aligned}
$$

Now let's start from the following:

$$
\begin{aligned}
& C_{2} Q_{j+1} \leq C_{3} \frac{r_{j+1}^{2} A_{j+1}-r_{j}^{2} A_{j}}{\left(r_{j+1}-r_{j}\right)^{2}}+C_{4}\left(Q_{j+1}-Q_{j}\right)^{\frac{1}{2}} \frac{\left(r_{j+1}^{2} A_{j+1}-r_{j}^{2} A_{j}\right)^{\frac{1}{2}}}{r_{j+1}-r_{j}} \\
& \leq C_{3} \frac{r_{j+1}^{2} A_{j+1}-r_{j}^{2} A_{j}}{\left(r_{j+1}-r_{j}\right)^{2}}+C_{4}\left\{\frac{\epsilon}{2}\left(Q_{j+1}-Q_{j}\right)+\frac{1}{2 \epsilon} \frac{r_{j+1}^{2} A_{j+1}-r_{j}^{2} A_{j}}{\left(r_{j+1}-r_{j}\right)^{2}}\right\} \\
& \leq\left(C_{3}+\frac{C_{4}}{2 \epsilon}\right) \frac{r_{j+1}^{2} A_{j+1}-r_{j}^{2} A_{j}}{\left(r_{j+1}-r_{j}\right)^{2}}+\frac{C_{4} \epsilon}{2}\left(Q_{j+1}-Q_{j}\right)
\end{aligned}
$$

where $\epsilon$ is sufficiently positive small. Choosing $\left\{r_{j}\right\}$ such that $r_{j+1} \geq 2 r_{j}$ (that is, $r_{j+1}-r_{j} \geq \frac{r_{j+1}}{2}$ ), we have:

$$
\frac{r_{j+1}^{2} A_{j+1}-r_{j}^{2} A_{j}}{\left(r_{j+1}-r_{j}\right)^{2}} \leq \frac{r_{j+1}^{2} A_{j+1}}{\left(\frac{r_{j+1}}{2}\right)^{2}}=4 A_{j+1}
$$

Furthermore, we have:

$$
\begin{aligned}
\left(C_{2}-\frac{C_{4} \epsilon}{2}\right) Q_{j+1} & \leq\left(C_{3}+\frac{C_{4}}{2 \epsilon}\right) \frac{r_{j+1}^{2} A_{j+1}-r_{j}^{2} A_{j}}{\left(r_{j+1}-r_{j}\right)^{2}} \\
& \leq 4\left(C_{3}+\frac{C_{4}}{2 \epsilon}\right) A_{j+1}
\end{aligned}
$$

where we chose $\epsilon$ to be sufficiently positive small enough such that $\left(C_{2}-\frac{C_{4} \epsilon}{2}\right)>0$. Via (13), for any strictly increasing sequence $\left\{r_{j}\right\}$ such that $r_{j+1} \geq 2 r_{j}$, we have $Q_{j} \rightarrow 0$ as $j \rightarrow \infty$ because $A_{j} \rightarrow 0$ by an assumption of $\liminf _{r \rightarrow \infty} \frac{1}{r^{2}} \int_{B(x ; r)}|\omega|^{q} d v=0$ for $q=2 m+2$. In addition, we notice that $\left.\left.|\omega|^{2 m-2}|\nabla| \omega\right|^{2}\right|^{2}=\left.\left.\frac{4}{(m+1)^{2}}|\nabla| \omega\right|^{m+1}\right|^{2}$. Therefore, we obtain the constancy property (i.e. $|\omega|=$ constant on $M$ ) due to the fact $\nabla|\omega|^{m+1} \equiv 0$ on $M$ by $Q_{j} \rightarrow 0$ as $j \rightarrow \infty$.

Next, we claim $|\omega|=0$. Otherwise, if $|\omega|=$ positive constant on $M$, by applying Poincaré-Sobolev Inequality (1) with $\varphi=\eta_{j}|\omega|^{m+1}$ for $\eta_{j}=\eta\left(x, r_{j}, r_{j+1}\right)$, via (12), we have:

$$
\begin{aligned}
S_{\alpha}|\omega|^{2 m+2}\left(\operatorname{vol}\left(B\left(r_{j}\right)\right)\right)^{1-\alpha} & \leq S_{\alpha}\left(\int_{M}\left|\left(\eta_{j}|\omega|^{m+1}\right)\right|^{\frac{2}{1-\alpha}} d v\right)^{1-\alpha} \\
& \leq \int_{M}\left|\nabla\left(\eta_{j}|\omega|^{m+1}\right)\right|^{2} d v \\
& =\int_{M}|\omega|^{2 m+2}\left|\nabla \eta_{j}\right|^{2} d v \\
& \leq \frac{C_{1}^{2}}{\left(r_{j+1}-r_{j}\right)^{2}} \int_{B\left(x, r_{j+1}\right) \backslash B\left(x, r_{j}\right)}|\omega|^{2 m+2} d v \\
& \leq C_{1}^{2} \frac{r_{j+1}^{2} A_{j+1}-r_{j}^{2} A_{j}}{\left(r_{j+1}-r_{j}\right)^{2}} \\
& \leq 4 C_{1}^{2} A_{j+1}
\end{aligned}
$$

If $|\omega|=$ positive constant, we have a contradiction because $S_{\alpha}|\omega|^{2 m+2}\left(\operatorname{vol}\left(B\left(r_{j}\right)\right)\right)^{1-\alpha}>0$ for any $j$ and $4 C_{1}^{2} A_{j+1} \rightarrow 0$ as $j \rightarrow \infty$ due to $A_{j} \rightarrow 0$ by an assumption of $\liminf _{r \rightarrow \infty} \frac{1}{r^{2}} \int_{B(x ; r)}|\omega|^{q} d v=0$ for $q=2 m+2$. Therefore, we obtain the vanishing property, which is $|\omega|=0$ leading to $\omega \equiv 0$.

In particular, $\omega \in L^{q}(M)$ (that is, $\int_{M}|\omega|^{q} d v<\infty$ ) must satisfy an assumption of $\liminf _{r \rightarrow \infty} \frac{1}{r^{2}} \int_{B(x ; r)}|\omega|^{q} d v=0$ for $q=2 m+2$. We can obtain the vanishing property for $\omega$ in $L^{q}$ space.

At the end, by combining (9) and $m \geq p \geq 2$, we compute the range of $q=2 m+2$ as follows:

$$
\max \left\{2 p+2, \frac{2\left(1-\sqrt{1-k_{\alpha} S_{\alpha}^{-1}}\right)}{k_{\alpha} S_{\alpha}^{-1}}\right\}<q<\frac{2\left(1+\sqrt{1-k_{\alpha} S_{\alpha}^{-1}}\right)}{k_{\alpha} S_{\alpha}^{-1}}
$$

Theorem 2 Let $M$ be a complete non-compact Riemannian manifold satisfying Poincaré-Sobolev Inequality (1) and Ricci $^{M} \geq-k(x)$ where $k(x) \geq 0$ is a continuous function such that

$$
k_{\alpha}: \triangleq\|k(x)\|_{L_{M}^{\frac{1}{\alpha}}}<S_{\alpha}
$$


Let $\omega$ be a closed and p-pseudo-coclosed differential 1-form on $M$ for $p \geq 2$. Then $\omega=0$ if $\omega$ has:

$$
\lim _{r \rightarrow \infty} \frac{\int_{\partial B\left(x_{0}, r\right)}|\omega|^{q} d v}{r}=0
$$

where $q$ is given by (15).

proof. For any strictly increasing sequence $\left\{r_{j}\right\}$ going to infinity such that $r_{j+1} \geq 2 r_{j}$ (that is $r_{j+1}-r_{j} \geq \frac{r_{j+1}}{2}$ ), via the first inequality of (13) and a definition of $G(r)=r^{2} A_{r}=\int_{B(r)}|\omega|^{2 m+2} d v$, we have:

$$
\begin{aligned}
\frac{C_{2}-\frac{C_{4} \epsilon}{2}}{C_{3}+\frac{C_{4}}{2 \epsilon}} Q_{j+1} & \leq \frac{G\left(r_{j+1}\right)-G\left(r_{j}\right)}{\left(r_{j+1}-r_{j}\right)^{2}} \\
& =\frac{G^{\prime}\left(r_{j}\right)}{r_{j+1}-r_{j}} \\
& \leq \frac{2 G^{\prime}\left(r_{j+1}\right)}{r_{j+1}}
\end{aligned}
$$

where we apply Mean Value Theorem for $G(r)$ with $\widetilde{r}_{j} \in\left[r_{j}, r_{j+1}\right]$ and observe that $G^{\prime}(r)=\int_{\partial B\left(x_{0}, r\right)}|\omega|^{2 m+2} d v$ is increasing by Coarea Formula. Letting $j \rightarrow \infty$, we have $Q_{j} \rightarrow 0$ by $\lim _{r \rightarrow \infty} \frac{G^{\prime}(r)}{r}=0$, which is equivalent to an assumption of $\lim _{r \rightarrow \infty} \frac{\int_{\partial B\left(x_{0}, r\right)}|\omega|^{q} d v}{r}=0$ for $q=2 m+2$. Then we prove the constancy property for $|\omega|$ by $Q_{j} \rightarrow 0$ as $j \rightarrow 0$ due to the same argument in Theorem 1. If $|\omega|$ is a positive constant, by applying Poincaré-Sobolev inequality (1) with $\varphi=\eta_{j}|\omega|^{m+1}$, via the similar argument in proof of (14), we have:

$$
S_{\alpha}|\omega|^{2 m+2}\left(\operatorname{vol}\left(B\left(r_{j}\right)\right)\right)^{1-\alpha} \leq C_{1}^{2} \frac{r_{j+1}^{2} A_{j+1}-r_{j}^{2} A_{j}}{\left(r_{j+1}-r_{j}\right)^{2}}
$$

On one hand, via (16), we know that $\frac{r_{j+1}^{2} A_{j+1}-r_{j}^{2} A_{j}}{\left(r_{j+1}-r_{j}\right)^{2}}=\frac{G\left(r_{j+1}\right)-G\left(r_{j}\right)}{\left(r_{j+1}-r_{j}\right)^{2}} \leq \frac{2 G^{\prime}\left(r_{j+1}\right)}{r_{j+1}} \rightarrow 0$ as $j \rightarrow \infty$. On the other hand, we know that $S_{\alpha}|\omega|^{2 m+2}\left(\operatorname{vol}\left(B\left(r_{j}\right)\right)\right)^{1-\alpha}>0$. Here we get a contradiction. Therefore, we obtain $|\omega| \equiv 0$ leading to $\omega \equiv 0$.

3.2 Liouville-type Results of Constancy Properties for p-Harmonic Maps

Lemma 2 Let $u: M \rightarrow N$ be a p-harmonic map. Let $\eta$ be a compactly supported non-negative smooth function on M, and $\varrho=\eta|d u|^{m}$. Then for $m \geq p \geq 2$, we have

$$
\begin{aligned}
& \int_{M} \varrho^{2}\langle\Delta d u, d u\rangle d v \\
& =\frac{(p-2)(2 m+2-p)}{4} \int_{M} \eta^{2}|d u|^{2 m-4}\left\langle d|d u|^{2}, d u\right\rangle^{2} d v+(p-2) \int_{M} \eta|d u|^{2 m-2}\left\langle d|d u|^{2}, d u\right\rangle\langle d \eta, d u\rangle d v \\
& \geq-\left.(p-2) \int_{M} \eta|\nabla \eta \| d u|^{2 m}|d| d u\right|^{2} \mid d v
\end{aligned}
$$

proof.

$$
\begin{aligned}
& \int_{M} \varrho^{2}\langle\Delta d u, d u\rangle d v \\
& =\int_{M} \eta^{2}|d u|^{2 m}\langle\Delta d u, d u\rangle d v \\
& =-\int_{M} \eta^{2}|d u|^{2 m}\left\langle d d^{*} d u, d u\right\rangle d v \\
& =-\int_{M} \eta^{2}|d u|^{2 m+2-p}\left\langle d d^{*} d u,|d u|^{p-2} d u\right\rangle d v \\
& =\int_{M}\left\langle-\eta^{2}|d u|^{2 m+2-p} d d^{*} d u,|d u|^{p-2} d u\right\rangle d v \\
& =\int_{M}\left\langle-d\left(\eta^{2}|d u|^{2 m+2-p} d^{*} d u\right)+d\left(\eta^{2}|d u|^{2 m+2-p}\right) d^{*} d u,|d u|^{p-2} d u\right\rangle d v \\
& =-\int_{M}\left\langle d\left(\eta^{2}|d u|^{2 m+2-p} d^{*} d u\right),|d u|^{p-2} d u\right\rangle d v+\int_{M}\left\langle d\left(\eta^{2}|d u|^{2 m+2-p}\right) d^{*} d u,|d u|^{p-2} d u\right\rangle d v \\
& =\int_{M}\left\langle\eta^{2}|d u|^{2 m+2-p} d^{*} d u,-d^{*}\left(|d u|^{p-2} d u\right)\right\rangle d v-\int_{M}\left\langle d\left(\eta^{2}|d u|^{2 m+2-p}\right)|d u|^{p-2}\left(-d^{*} d u\right), d u\right\rangle d v \\
& =-\int_{M}\left\langle d\left(\eta^{2}|d u|^{2 m+2-p}\right)|d u|^{p-2} \tau(u), d u\right\rangle d v \\
& =-\int_{M}\left\langle\left\langle 2 \eta d \eta|d u|^{2 m+2-p}+(2 m+2-p) \eta^{2}|d u|^{2 m+1-p} d|d u|\right\}|d u|^{p-2} \tau(u), d u\right\rangle d v \\
& =\int_{M}(p-2)|d u|^{p-3}\langle d|d u|, d u\rangle\left\{2 \eta|d u|^{2 m+2-p}\langle d \eta, d u\rangle+(2 m+2-p) \eta^{2}|d u|^{2 m+1-p}\langle d|d u|, d u\rangle\right\} d v \\
& =\int_{M} 2(p-2) \eta|d u|^{2 m-1}\langle d|d u|, d u\rangle\langle d \eta, d u\rangle d v+\int_{M}(p-2)(2 m+2-p) \eta^{2}|d u|^{2 m-2}\langle d|d u|, d u\rangle^{2} d v \\
& =\int_{M}(p-2) \eta|d u|^{2 m-2}\left\langle d|d u|^{2}, d u\right\rangle\langle d \eta, d u\rangle d v+\int_{M} \frac{(p-2)(2 m+2-p)}{4} \eta^{2}|d u|^{2 m-4}\left\langle d|d u|^{2}, d u\right\rangle^{2} d v
\end{aligned}
$$

where we have used the facts

$$
\triangle d u=-\left(d d^{*}+d^{*} d\right) d u, \quad-d^{*} d d u=0
$$

in the second step; and

$$
-\eta^{2}|d u|^{2 m+2-p} d d^{*} d u=-d\left(\eta^{2}|d u|^{2 m+2-p} d^{*} d u\right)+d\left(\eta^{2}|d u|^{2 m+2-p}\right) d^{*} d u
$$


due to the product rule of

$$
d\left(\eta^{2}|d u|^{2 m+2-p} d^{*} d u\right)=\eta^{2}|d u|^{2 m+2-p} d d^{*} d u+d\left(\eta^{2}|d u|^{2 m+2-p}\right) d^{*} d u
$$

in the fifth step; and by switching an operator of $d$ on one side to its adjoint operator of $d^{*}$ on the other side in the seventh step; and applying a $p$-tension field of $u$ as well as a 2-tension field of $u$

$$
\tau_{p}(u)=-d^{*}\left(|d u|^{p-2} d u\right)=0, \quad \tau(u)=-d^{*} d u
$$

in the eighth step; and the identity between $\tau_{p}(u)$ and $\tau(u)$

$$
|d u|^{p-2} \tau(u)=-(p-2)|d u|^{p-3}\langle d|d u|, d u\rangle
$$

due to

$$
0=\tau_{p}(u)=(p-2)|d u|^{p-3}\langle d|d u|, d u\rangle+|d u|^{p-2} \tau(u)
$$

in the tenth step; and

$$
d|d u|=\frac{d|d u|^{2}}{2|d u|}
$$

in the last step.

Theorem 3 Let $u$ be a p-harmonic map for $p \geq 2$ from the domain $M$ to the target space $N$. Suppose the domain manifold $M$ is a complete non-compact Riemannian manifold satisfying the Poincaré-Sobolev Inequality (1) and Ricci ${ }^{M} \geq-k(x)$ where $k(x) \geq 0$ is a continuous function such that

$$
k_{\alpha}: \triangleq\|k(x)\|_{L_{M}^{\frac{1}{\alpha}}}<S_{\alpha}
$$

Suppose the target space $N$ is a Riemannian manifold with Riem $^{N} \leq 0$. Then $u$ is constant if du has

$$
\liminf _{r \rightarrow \infty} \frac{1}{r^{2}} \int_{B(x ; r)}|d u|^{q} d v=0
$$

In particular, $u$ is constant if $d u \in L^{q}(M)$ has

$$
\int_{M}|d u|^{q} d v<\infty
$$

And $q$ is given by (15).

proof. By Weitzenböck Bochner Formula, we have:

$$
\begin{aligned}
& \frac{1}{2} \Delta|d u|^{2} \\
= & \langle\Delta d u, d u\rangle+|\nabla d u|^{2}+\sum_{j=1}^{n}\left\langle d u\left(\operatorname{Ricci}^{M}\left(e_{j}\right)\right), d u\left(e_{j}\right)\right\rangle-\sum_{i, j=1}^{n}\left\langle\operatorname{Riem}^{N}\left(d u\left(e_{j}\right), d u\left(e_{i}\right)\right) d u\left(e_{i}\right), d u\left(e_{j}\right)\right\rangle \\
\geq & \langle\Delta d u, d u\rangle+|\nabla d u|^{2}-k(x)|d u|^{2}
\end{aligned}
$$

For the test function $\varrho=\eta|d u|^{m}$, via Lemma 2, this follows immediately from the proof of Theorem 1 by setting $\omega=d u$. We obtain the constancy property of $u$ by $d u \equiv 0$.

Theorem 4 Let $u$ be a p-harmonic map for $p \geq 2$ from the domain manifold $M$ to the target space $N$. Suppose the domain manifold $M$ is a complete non-compact Riemannian manifold satisfying the Poincaré-Sobolev Inequality (1) and Ricci $^{M} \geq-k(x)$ where $k(x) \geq 0$ is a continuous function such that

$$
k_{\alpha}: \triangleq\|k(x)\|_{L_{M}^{\frac{1}{\alpha}}}<S_{\alpha}
$$

Suppose the target space $N$ is a Riemannian manifold with Riem $^{N} \leq 0$. Then $u$ is constant if du has

$$
\lim _{r \rightarrow \infty} \frac{\int_{\partial B\left(x_{0} ; r\right)}|d u|^{q} d v}{r}=0
$$

where $q$ is given by (15). 
proof. By Weitzenböck Bochner Formula, we have:

$$
\begin{aligned}
& \frac{1}{2} \Delta|d u|^{2} \\
= & \langle\Delta d u, d u\rangle+|\nabla d u|^{2}+\sum_{j=1}^{n}\left\langle d u\left(\operatorname{Ricci}^{M}\left(e_{j}\right)\right), d u\left(e_{j}\right)\right\rangle-\sum_{i, j=1}^{n}\left\langle\operatorname{Riem}^{N}\left(d u\left(e_{j}\right), d u\left(e_{i}\right)\right) d u\left(e_{i}\right), d u\left(e_{j}\right)\right\rangle \\
\geq & \langle\Delta d u, d u\rangle+|\nabla d u|^{2}-k(x)|d u|^{2}
\end{aligned}
$$

For the test function $\varrho=\eta|d u|^{m}$, via Lemma 2, this follows immediately from the proof of Theorem 2 by setting $\omega=d u$. We obtain the constancy property of $u$ by $d u \equiv 0$.

\section{Discussion}

Liouville-type problems are solved with two significant technical breakthroughs in this article. The first breakthrough is an extension from manifolds with only one sign of curvatures to manifolds with the mixed signs of curvatures. The second breakthrough is a generalization from finite $q$-energy to infinite $q$-energy. To overcome obstacles of an extension on manifolds, the technique of Poincaré-Sobolev Inequality is applied to widen the scopes of manifold structures from one sign of curvatures to the mixed signs of curvatures. To overcome obstacles of an extension about $q$-energy, the technique of $p$-balanced growth is used to generalize $q$-energy growth from finite to infinite. These two technical breakthroughs are illustrated in an innovative computational method. This new way of computing in calculation reveals interactions among changes of power in a test function, changes of manifold structures, and changes of energy growth rates. On one hand, the maximum scope of energy growth rates is indicated by the maximum range of power in the test function. On the other hand, the maximum scope of energy growth rates should be compatible with the maximum scope of manifold structures, which are supported by Poincaré-Sobolev Inequality. Weitzenböck Bochner Formula, Poincaré-Sobolev Inequality, Hölder Inequality, Cauchy-Schwarz Inequality, and Calculus skills as estimation techniques have been presented in calculation. Liouville-type problems on manifolds with the mixed curvatures signs approaching to infinite $q$-energy have been solved. Liouville-type results such as vanishing properties for differential forms and constancy properties for $p$-harmonic maps have been obtained on manifolds with Poincaré-Sobolev Inequality in the context of $p$-balanced energy growth.

The research work in this article is to study Liouville-type Theorems in the general settings against constraints from curvature signs on manifolds and restrictions from $q$-energy growth for differential forms or differential maps. In the future, we would like to continue to study the more complicated differential forms such as vector-valued forms than the simple case of differential 1-forms. In addition, we could pay attentions to $F$-harmonic maps with $F$-energy, which are the generalization of $p$-harmonic maps with $p$-energy where $F$ means a function and $p$ means a number. It turns out that the limited manifold structures hold back the limited energy growth rates for differential forms or differential maps. We would like to be interested in a wide variety of manifold structures such as pseudo-Riemannian manifolds. In summary, three potential follow-up research directions could be considered:

1. Liouville-type problems for differential forms such as vector-valued forms

2. Liouville-type problems for differential maps such as $F$-harmonic maps with $F$-energy

3. Liouville-type problems on manifolds with special structures such as pseudo-Riemannian-manifolds

Estimation techniques in the context of $p$-balanced energy growth could be applied to investigate Liouville-type problems in future research.

\section{Acknowledgements}

Research was supported by Faculty Development Grant at Borough of Manhattan Community College-The City University of New York.

\section{References}

Cheung, L.-F., \& Leung, P.-F. (1995). A remark on convex functions and p-harmonic maps. Geometriae Dedicata, 56(3), 269-270. https://doi.org/10.1007/bf01263568

Greene, R., \& Wu, H. (1981). Harmonic forms on noncompact Riemannian and Kahler manifolds. The Michigan Mathematical Journal, 28(1), 63-81. https://doi.org/10.1307/mmj/1029002458

Kawai, S. (1994). p-Harmonic maps and convex functions. Geometriae Dedicata, 74(3), 261-265. https://doi.org/10.1023/a:1005042831356 
Pigola, S., Rigoli, M., \& Setti, A. G. (2008). Constancy of p-harmonic maps of finite $q$-energy into non-positively curved manifolds, Mathematische Zeitschrift, 258(2), 347-362. https://doi.org/10.1007/s00209-007-0175-7

Schoen, R., \& Yau, S. T. (1976). Harmonic maps and topology of stable hypersurfaces and manifolds with non-negative Ricci curvature. Comment. Math. Helv., 51(1), 333-341. https://doi.org/10.1007/bf02568161

Wei, S. W., Li, J., \& Wu, L. (2008). Generalizations of the uniformization theorem and Bochner's method in p-harmonic geometry. Commun. Math. Anal., Conf., 1, 46-68.

Wei, S. W., \& Wu, L. (2015). Vanishing theorems for 2-balanced harmonic forms. Global Journal of Pure and Applied Mathematics, 11(2), 745-753. http://www.ripublication.com/gjpam.htm

Wu, L., \& Li, Y. (2016). Generalizing Liouville-type problems for differential 1-forms from $L^{q}$ spaces to non- $L^{q}$ spaces. International Journal of Mathematical Analysis, 10(28), 1375-1387. https://doi.org/10.12988/ijma.2016.610117

Zhang, X. (2001). A note on p-harmonic 1-form on complete manifolds. Canad. Math. Bull., 44(3), 376-384. https://doi.org/10.4153/CMB-2001-038-2

\section{Copyrights}

Copyright for this article is retained by the author(s), with first publication rights granted to the journal.

This is an open-access article distributed under the terms and conditions of the Creative Commons Attribution license (http://creativecommons.org/licenses/by/4.0/). 